\title{
Interferon- $\alpha$ als Therapieoption bei der Behandlung des schwergradig persistierenden, unkontrollierten Asthma bronchiale: Eine offene Studie
}

\author{
Interferon- $\alpha$ as Treatment Option in Severe Persistent Uncontrolled Bronchial \\ Asthma: An Open Label Study
}

Autoren

Institute
C. Kroegel, N. Bergmann, C. Heider, A. Moeser, J. Happe, Y. Schlenker, B. Bartuschka, M. Henzgen, R. Walther, A. Reißig, M. Foerster

Pneumologie \& Allergologie/Immunologie, Klinik für Innere Medizin I, Friedrich-Schiller-Universität, Jena eingereicht 18.12 .2008 akzeptiert nach Revision 4. 5.2009

\section{Bibliografie}

DOI $10.1055 / \mathrm{s}-0029-1214738$ Pneumologie 2009; 63: 307-313 @ Georg Thieme Verlag KG Stuttgart · New York ISSN 0934-8387

\section{Korrespondenzadresse} Prof. Dr. med. Claus Kroegel Pneumologie \& Allergologie/ Immunologie Innere Medizin I Friedrich-Schiller-Universität Erlanger Allee 101 07740 Jena claus.kroegel@med.uni-jena.de

\section{Zusammenfassung \\ $\nabla$}

Hintergrund: Die Studie untersucht die therapeutische Wirkung einer immunmodulatorischen Therapie mit IFN-alpha (IFN- $\alpha$ ) bei Patienten mit kortikosteroidabhängigem, schwergradig persistierendem, unkontrolliertem Asthma bronchiale. Patienten und Methodik: 16 Pat. (14 w/2 m, Alter 39 Jahre [24-63]) erfüllten die Kriterien der Deutschen Atemwegsliga für ein Asthma bronchiale im Schweregrad IV. 8 Pat. beendeten die Therapie innerhalb von 7 Monaten (M) (Nebenwirkungen: 3, abgelehnte Kostenübernahme: 2, Non-Compliance: 2, Wohnortwechsel: 1). Die übrigen 8 Pat. (8 w) im Alter von 49 (35 - 68) Jahren (Krankheitsdauer 16 (5-24) Jahre) erhielten IFN- $\alpha(9 \mu \mathrm{g}) 3 \times$ wöchentlich s.c. über mindestens 12 M. Alle Patienten erhielten orale Glukokortikoiden (GCs) seit über 5 Jahren (durchschnittliche Dosis 17,5 [5,0-64,0] mg/d). Klinik, Lungenfunktion, Differenzialblutbild, Bedarf an $\beta_{2}$-Mimetika und Zahl der Notarztbehandlungen sowie Hospitalisierungen und das Patiententagebuch wurden vor und 12 Monate nach Therapiebeginn ermittelt. Die Daten sind als \% des Solls bzw. Median [Minimum-Maximum] angegeben.

Ergebnisse: IFN- $\alpha$ führte innerhalb von $12 \mathrm{M} \mathrm{zu}$ einer Verbesserung der Lungenfunktion: FEV1 64 vs $75 \%$, Tiffenau-Indexes 76 vs $89 \%$, RV $153 \%$ vs $129 \%$, Rtot 193 vs $111 \%$ und des PEF-Wertes um 50-190 L/min. Darüber hinaus verringerte sich der Bedarf an $\beta_{2}$-Mimetika/d (10 [2-20] vs. 1 [0 - 3] Hub/d), die Zahl der gestörten Nächte/M (11 [4-30] vs. 1 [0 - 5]), die Zahl der Notarztinterventionen/M ( 7 [2-15] vs. 0 [0-5]) sowie der Hospitalisierungen/Jahr (4 [1 - 8] vs. 0 [0-5]). Bei 5 Patienten verschwanden die Asthmaanfälle und gestörten Nächte völlig. Die Kortisondosis konnte von 17,5 (5,0-64,0) auf $2(0-16) \mathrm{mg} / \mathrm{d}$ reduziert werden. Bei 5 Pat. (62,5\%) konnte die orale Kortisontherapie beendet werden. Die Bluteosinophilenzahl nahm von 0,46 auf 0,28 Gpt/L ab. Passage-

\section{Abstract \\ $\nabla$}

Background: The purpose of this study was to evaluate the long-term safety and therapeutic effects of IFN- $\alpha$ in patients with severe persistent uncontrolled asthma on long-term oral glucocorticoid (GC) treatment.

Patients and Methods: The study included 16 patients ( 2 male, 14 female; age 39 years [range: 24-63]) with severe persistent asthma. Diagnosis and severity classification of asthma were established according to the guidelines of the „Deutsche Atemwegsliga“. Eight patients stopped the therapy within 7 months due to side effects $(n=3)$, costs not covered by health insurance $(\mathrm{n}=2)$, non-compliance $(\mathrm{n}=2)$, and change of residence $(n=1) .8$ patients ( 8 female, age 49 years [range: $35-68$ ], duration of disease 16 years [range: 5-24]) were treated for at least 12 months with IFN- $\alpha(9 \mu \mathrm{g}) 3$ times/week. All patients were on oral glucocorticoids (GCs) for more than 5 years (average dose 17.5 [range: $5.0-64.0] \mathrm{mg} / \mathrm{d}$ ). Clinical signs, lung function, need for reliever medication, number of emergency visits and hospitalisations and diary were assessed prior to and after 12 months of treatment. Data are given as percent of normal or median [range].

Results: IFN- $\alpha$ improved lung function after 12 months: FEV1 64 vs. 75\%; FEV1/IVC 76 vs. 89\%; RV $153 \%$ vs. $129 \%$; Rtot 193 vs. $111 \%$ and morning PEF by $50-190 \mathrm{~L} / \mathrm{min}$. IFN- $\alpha$ also significantly reduced the use of reliever medication $(10$ [2-20] vs. 1 [0-3] puffs/d), nocturnal awakening (11 [4-30] vs. $1[0-5] /$ month), emergency visits (7 $[2-15]$ vs. $0[0-5] /$ month) and hospitalisations $(4[1-8]$ vs. $0[0-5] /$ year). In 5 patients the asthma attacks and nightly disturbances disappeared completely. The improvements were achieved despite a tapering of the oral GCs in all patients from $17.5(5.0-64.0)$ to $2(0-16) \mathrm{mg} / \mathrm{d}$. In 5 patients GC treatment could be discontinued. The number of blood eosinophils decreased from 0.46 to 
re Nebenwirkungen: allgemeines Krankheitsgefühl, Arthralgie, Übelkeit. Zwei Pat. entwickelten eine Autoimmunthyreoiditis.

Schlussfolgerung: Die Therapie des kortisonabhängigen, schwergradig persistierenden Asthma bronchiale mit IFN- $\alpha$ über 12 Monate führt zu einer anhaltenden Verbesserung der Krankheitskontrolle und erlaubt eine Reduktion bzw. Beendigung der langjährigen oralen Kortisontherapie. Schwere Nebenwirkungen können in Einzelfällen auftreten.

\section{Einleitung}

Die Mehrzahl der Patienten mit persistierendem Asthma leidet an einer leichten bis moderaten Form der Erkrankung, die sich durch die regelmäßige Therapie mit anti-entzündlichen und bronchialerweiternden Medikamenten weitgehend kontrollieren lässt [1]. Eine kleine Untergruppe von bis zu 1\% der Asthmatiker bleibt jedoch trotz Einsatz aller derzeit verfügbaren therapeutischen Möglichkeiten symptomatisch [2,3]. Bei diesem schwergradig persistierenden Asthma handelt es sich um eine Form mit ununterbrochenen Krankheitsbeschwerden (Dyspnoe, Husten), die sich gleichermaßen tagsüber wie nachts äußern und den Betroffenen das Führen eines „normalen“ Lebens ebenso wie einen erholsamen Schlaf unmöglich machen. Bereits geringste Anforderungen des täglichen Lebens, wie An- und Auskleiden oder Zähneputzen, werden zu einer Herausforderung, die regelmäßig von Husten- und Dyspnoefällen begleitet wird. Andere Tätigkeiten (Einkaufen, Treppensteigen, Putzen, Kochen) können im Einzelfall gar nicht erst von den Betroffenen bewältigt werden. Hinzu kommen häufig auftretende, z.T. schwere Asthmaexazerbationen, die oft einen Notarztbesuch oder eine Hospitalisierung erforderlich machen.

Aufgrund des Fehlens einer wirkungsvollen Therapie besteht ein Bedarf an Medikamenten, mit denen sich das Asthma der schwersten Verlaufsform besser kontrollieren lässt. Ziel dieser Studie ist deshalb, die therapeutische Wirkung von Interferon- $\alpha$ bei der Behandlung des schwergradig persistierenden, unkontrollierten Asthma bronchiale im Hinblick auf klinische, lungenfunktionelle und laborchemische Kriterien zu untersuchen.
$0.28 \mathrm{Gpt} / \mathrm{L}$. Adverse events were transient and usually decreased within 3 to 4 weeks. Two patients developed an autoimmune thyreoiditis.

Conclusion: In severe persistent, uncontrolled, and GC-dependent asthma, treatment with IFN- $\alpha$ leads to sustained clinical improvement and allows the reduction or discontinuation of oral GCs. Severe side effects may occur in isolated cases.

\section{Patienten, Material und Methoden}

\section{Patientencharakteristika}

Für die Studie wurden Patienten mit schwergradig persistierendem Asthma bronchiale (Schweregrad IV) ausgewählt. Ausschlusskriterien waren Raucheranamnese, Einnahme von $\beta$-Blockern oder ACE-Hemmern sowie das Vorliegen von Kontraindikationen für Inferax ${ }^{\circledR}$ (Leukozytopenie, Thrombopenie, fortgeschrittene Leberzirrhose, zerebrale Krampfleiden, bestehende Depressionen oder Schwangerschaft). Zwischen November 1999 und Juli 2003 wurden 16 Patienten (2 Männer, 14 Frauen; Alter 50 [26-68] Jahre) mit einer Erkrankungsdauer im Median von 16 (5 - 24) Jahren aufgenommen ( Tab. 1).

Alle Patienten litten an schwergradig persistierendem Asthma bronchiale, erfüllten die diagnostischen Kriterien der Deutschen Atemwegsliga und wurden mehr als 5 Jahre mit oralen Glukokortikoiden $(20[5-65] \mathrm{mg} / \mathrm{d})$ therapiert. Zusätzlich setzten sie inhalative kurzwirksame $\beta_{2}$-Mimetika mehrfach täglich (10 [2-20] Hübe/d) als Bedarfsspray ein. Die Patienten klagten über regelmäßige Störungen des Nachtschlafes, Einschränkungen der körperlichen Leistungsfähigkeit und bedurften mindestens einer Notarztbehandlung/Monat.

Für die Studie wurden insgesamt 8 weibliche Patienten (Alter 49 [35-68] Jahre) mit schwergradig persistierendem Asthma bronchiale über einen Beobachtungszeitraum von 12 Monaten unter Interferon- $\alpha$-Therapie betrachtet. Die Studie wurde von der lokalen Ethikkommission genehmigt. Die übrigen 8 von den $16 \mathrm{~Pa}-$ tienten beendeten die Therapie innerhalb von 7 Monaten (M) (Nebenwirkungen: 3, abgelehnte Kostenübernahme: 2, NonCompliance: 2, Wohnortwechsel: 1) ( Tab. 1).

\begin{tabular}{|c|c|c|c|c|}
\hline Patient & Alter & Geschlecht & Erstdiagnose Asthma & Abbruchgründe (Dauer der Therapie) \\
\hline 1 & 64 & w & 1983 & keine signifikanten Nebenwirkungen \\
\hline 2 & 35 & w & 1996 & keine signifikanten Nebenwirkungen \\
\hline 3 & 44 & w & 1993 & keine signifikanten Nebenwirkungen \\
\hline 4 & 54 & w & 1984 & keine signifikanten Nebenwirkungen \\
\hline 5 & 68 & w & 1984 & keine signifikanten Nebenwirkungen \\
\hline 6 & 40 & w & 1978 & keine signifikanten Nebenwirkungen \\
\hline 7 & 67 & w & 1979 & keine signifikanten Nebenwirkungen \\
\hline 8 & 35 & w & 1993 & keine signifikanten Nebenwirkungen \\
\hline 9 & 65 & $\mathrm{~m}$ & 1997 & $\begin{array}{l}\text { Hyperemesis, Fieber, Abgeschlagenheit } \\
\text { (4 Monate) }\end{array}$ \\
\hline 10 & 54 & w & 1987 & keine Kostenübernahme (7 Monate) \\
\hline 11 & 41 & w & 1989 & $\begin{array}{l}\text { anhaltende Abgeschlagenheit, } \\
\text { depressive Stimmungslage (14 Tage) }\end{array}$ \\
\hline 12 & 34 & w & 1979 & Non-Compliance (6 Monate) \\
\hline 13 & 54 & $\mathrm{~m}$ & 1978 & Non-Compliance (6 Monate) \\
\hline 14 & 45 & w & 1997 & $\begin{array}{l}\text { anhaltende Abgeschlagenheit, Übelkeit, } \\
\text { depressive Stimmungslage ( } 1 \text { Monat) }\end{array}$ \\
\hline 15 & 26 & w & 1996 & Wohnortwechsel \\
\hline 16 & 56 & w & 1980 & keine Kostenübernahme (2 Monate) \\
\hline
\end{tabular}

Tab. 1 Charakterisierung der Patienten. 

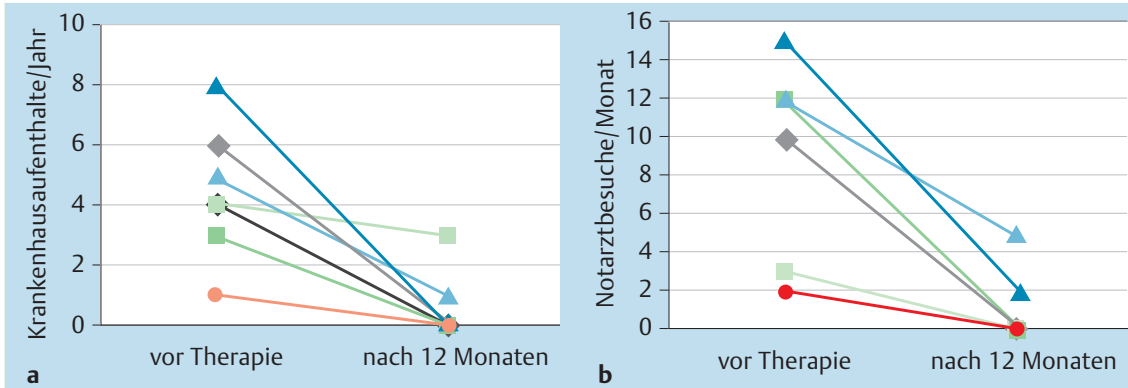

Abb. 1 Vergleich der Häufigkeit ärztlicher Interventionen und Symptome vor und nach 12 Monaten Behandlung mit IFN- $\alpha$ bei Patienten mit schwergradig persistierendem Asthma bronchiale. Es wurde die Anzahl der Krankenhausaufenthalte/Jahr (a), die Anzahl der Notfallbehandlungen/Monat (b), die Asthmaanfallshäufigkeit/Tag (c) sowie die Anzahl nächtlicher Dyspnoeanfälle/Monat (d) für Patienten $(n=8)$ mit schwergradig persistierendem Asthma bronchiale Schweregrad IV aus dem Patientenfrageb bogen ermittelt.
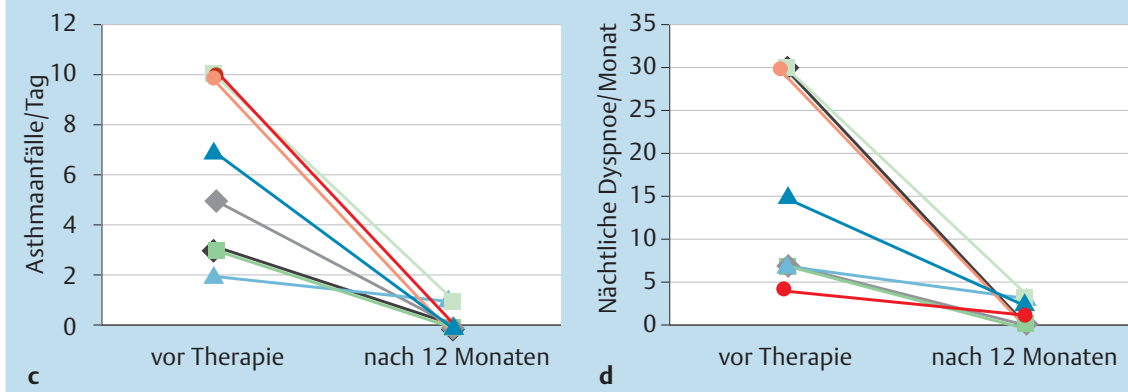

\section{IFN- $\alpha$-Therapie}

Die Therapie basierte auf der wöchentlichen subkutanen Gabe von $3 \times 9 \mu$ Interferon- $\alpha$ con (Inferax ${ }^{\circledR}$ Yamanouchi, Deutschland). Die erste Interferon-Gabe erfolgte unter stationären Bedingungen. Zur Überwachung der Nebenwirkungen von Interferon$\alpha$ erfolgten regelmäßige Kontrollen des Blutbildes, der Schilddrüsenparameter sowie der Leber- und Nierenwerte. Die Daten zur Bestimmung des Therapieeffektes wurden vor Therapiebeginn und nach 12 Monaten Behandlung erhoben.

\section{Patientenfragebogen}

Kontrolle und Monitoring des subjektiven Befindens erfolgte mittels Fragebogen und persönlicher Befragung zur Häufigkeit von Asthmaanfällen/Tag, Bedarfsmedikation/Tag, nächtliche Dyspnoeanfälle/Woche, Hustenanfälle/Tag, Krankenhausaufenthalte/Jahr, Notarztbesuche/Monat zu den festgelegten Messzeitpunkten.

\section{Lungenfunktion}

Vor Beginn der Studie und nach 12 Monaten Behandlung erfolgte die Lungenfunktionsprüfung mittels Ganzkörperbodyplethysmographie (Jäger Masterscreen Body, Hoechberg, Deutschland).

\section{Zytologie und Laborparameter}

Die Anzahl und Verteilung der Blutzellen sowie deren Subpopulationen wurden mittels eines Standardhämatometers zu den einzelnen Messzeitpunkten bestimmt. Die zytologische Auswertung der Blutzellen erfolgte nach Zytozentrifugation und anschließender Färbung (May-Grünwald-Giemsa-Färbung) per Hand unter einem Lichtmikroskop (Zeiss, Axiolab, Jena, Deutschland). Dabei wurde der relative Anteil der Leukozyten-Subpopulationen aufgrund einer differentiellen Auswertung von 200 Zellen unter dem Lichtmikroskop von einem erfahrenen Zytologen ausgezählt. Andere Parameter umfassten: TSH, T3, T4, r-GT, LDH, Lipase, C-reaktives Protein (CRP) und Blutkörperchensenkgeschwindigkeit als Entzündungsmarker.

\section{Statistik}

Die statistische Auswertung der erhobenen Daten erfolgte mittels Excel 2003 und SPSS 10.0 für Windows. Die Messwerte sind als Median dargestellt. Im Text sind zusätzlich Minimum und Maximum (Bereich) angegeben. Die Beschreibung der Streuung erfolgt in den Abbildungen durch den Interquartileabstand. Für die Berechnung von Unterschieden innerhalb der Patientengruppe wurde der Wilcoxon-Test für verbundene, nicht-normalverteilte Stichproben gewählt. Eine Irrtumswahrscheinlichkeit von $\mathrm{p}<0,05$ wurde als statistisch signifikant gewertet.

\section{Ergebnisse}

\section{Krankheitskontrolle}

Bei den Patienten konnte ein signifikanter Rückgang der Krankenhausaufenthalte von 4,0 $(1,0-8,0)$ auf $0,0(0,0-3,0)$ pro Jahr im Therapiezeitraum von 12 Monaten verzeichnet werden $(p<0,05)$. Bei 6 Patienten war nach Therapieeinleitung keine stationäre Behandlung mehr aufgrund von Exazerbationen im Betrachtungszeitraum erforderlich $(\boldsymbol{\bullet} \mathbf{A b b} \mathbf{1} \mathbf{1} \mathbf{a})$.

Auch für die verbleibenden 2 Asthmatiker war die Anzahl der Krankenhausaufenthalte pro Jahr rückläufig. In gleicher Weise nahm die Anzahl der Notarztinterventionen pro Monat ( $\bullet$ Abb. 1 b ) signifikant von 6,5 (2,0-15,0) auf 0,0 (2,0-5,0) Notarztbesuche pro Monat $(\mathrm{p}<0,05)$ ab. Nach 12 Monaten konnten unter Interferon- $\alpha$-Therapie 6 Patienten vollständig auf Notarztbesuche verzichten. Auch für die Häufigkeit von Asthmaattacken ließ sich eine signifikante Abnahme von $6(2-10)$ auf $0(0-3)$ pro Tag $(p<0,05)$ unter Interferon- $\alpha$-Therapie nach 12 Monaten nachweisen $(\bullet$ Abb.1c). Die Zahl nächtlicher Dyspnoe- oder Hustenanfälle mit Unterbrechung des Schlafes nahm signifikant von 11,0 (4,0-30,0) auf 0,5 (0,0-3,0) pro Monat ( $\bullet$ Abb. 1 d) ab.

\section{Peak-Flow}

In der $\odot$ Tab. 2 sind die PEF-Bestwerte der Patienten $(n=8)$ in $1 /$ min vor Therapie den Bestwerten unter IFN- $\alpha$-Therapie gegenübergestellt. 
Tab. 2 Peak-Flow-Veränderung unter Interferon- $\alpha$-Therapie. Dargestellt sind die PEF-Bestwerte in I/min vor Therapie, unter Therapie sowie die Differenz der PEF-Bestwerte. Zusätzlich sind Median und Bereich ausgewiesen. Die Daten wurden auf Grundlage der Asthma-Tagebücher und Patientenfragebögen für 8 Asthmatiker ermittelt.

\begin{tabular}{|c|c|c|c|}
\hline \multirow[b]{2}{*}{ Patient } & \multicolumn{3}{|c|}{ PEF-Bestwert [I/min] } \\
\hline & Vor Therapie & Unter Therapie & Differenz \\
\hline 1 & 340 & 430 & 90 \\
\hline 2 & 300 & 400 & 100 \\
\hline 3 & 150 & 340 & 190 \\
\hline 4 & 300 & 400 & 100 \\
\hline 5 & 300 & 350 & 50 \\
\hline 6 & 100 & 250 & 150 \\
\hline 7 & 150 & 200 & 50 \\
\hline 8 & 350 & 400 & 50 \\
\hline Median & $300(100-350)$ & $375(200-430)$ & $95(50-190)^{*}$ \\
\hline
\end{tabular}

Auf der Grundlage der Asthma-Tagebücher der Patienten wurde der PEF in $1 /$ min vor und unter Therapie ermittelt. Dabei kam es unter der Behandlung mit Interferon- $\alpha$ zu einer signifikanten Zunahme des PEF von 300 (100-350) 1/min auf 375 (200-430) 1/min.

FEV $_{1}$

Die $\mathrm{FEV}_{1}$ zeigte unter Therapie nach 12 Monaten Behandlung eine tendenzielle Zunahme von 64,2\% (31,4-90,8) auf 75,4\% (41,5 - 108,2) ( $\bullet$ Abb. 2 a).

Für vier Patienten konnte eine kontinuierliche Verbesserung und Stabilisierung der $\mathrm{FEV}_{1}$-Werte über den gesamten Therapiezeitraum verzeichnet werden. Davon normalisierte sich bei zwei Patienten nach 12 Monaten Interferon- $\alpha$-Therapie das $\mathrm{FEV}_{1}$. Die verbleibenden zwei Patienten erzielten eine Zunahme des FEV von $30 \%$. Ein Patient mit einer initial sehr stark eingeschränkten $\mathrm{FEV}_{1}$ (31,0\% des Solls) zeigte im Verlauf variable FEV $_{1}$-Werte zwischen $41 \%$ und $73 \%$. Bei drei Patienten nahm die $\mathrm{FEV}_{1}$ gegenüber dem Ausgangsniveau leicht ab. Diese drei Patienten (3, 6, 7) litten zum Messzeitpunkt 12 Monate an einem bronchopulmonalen Infekt ( Abb.2a).

\section{Tiffenau-Index}

Der Tiffenau-Index zeigte eine ansteigende Tendenz von 76,7\% $(58,0-96,0)$ auf $89,1 \%(65,1$ - 94,0) ( Abb. 2 b). Bei fünf Patienten stieg der Tiffenau-Index unter IFN- $\alpha$-Therapie kontinuierlich an $($ Abb. 2 b). Bei diesen Patienten war eine 7-20\%ige Verbesserung des Tiffenau-Indexes zu beobachten. Drei Patienten $(1,6)$, mit fast normalen $\mathrm{FEV}_{1}$ /VC-Quotienten unter Kortisontherapie vor Therapie (96,0\% und 91,6\%) zeigten keine Änderungen (94,0\% und 93,7\% nach 12 Monaten Behandlung).

\section{RV}

Das Residualvolumen nahm von 153,0\% (88,0-164,3) vor Therapieeinleitung auf $129,4 \%(84,9-203,9)$ nach 12 Monaten IFN- $\alpha-$ Behandlung ab ( $\bullet$ Abb. 2c c). Bei zwei Patienten mit Infekt $(3,7)$ war eine geringe Zunahme des Residualvolumens zu verzeichnen.

$\mathbf{R}_{\text {AW }}$

Unter der Interferon- $\alpha$-Therapie kam es zu einer deutlichen Reduktion des $\mathrm{R}_{\mathrm{AW}}$ von 193,7\% (102,0 - 497,6) vor Therapiebeginn auf $111,6 \%(67,3-356,5)$ nach 12 Monaten IFN- $\alpha$-Behandlung. Ein Patient mit Infekt (7) zeigte eine Zunahme des Atemwegswiderstandes im Rahmen eines Infektes ( $\bullet$ Abb. $2 \mathbf{d})$.

\section{Glukokortikoidtherapie}

Vor Therapie lag der Median der oralen Glukokortikoiddosis bei 17,5 (5,0-64,0) mg/d. Unter Therapie war eine Reduktion des Einsatzes von Glukokortikoiden möglich $(\mathrm{p}<0,05)$. Nach 12 Monaten Therapie lag die orale Glukokortikoiddosis bei $2,0(0,0-$ $16,0) \mathrm{mg} / \mathrm{d}$. Bei fünf Patienten $(1,2,4,7,8)$ war es innerhalb des Studienzeitraumes möglich, die orale Glukokortikoidtherapie zu beenden ( Abb.3a). Bei den übrigen Patienten ließ sich die Dosis reduzieren.

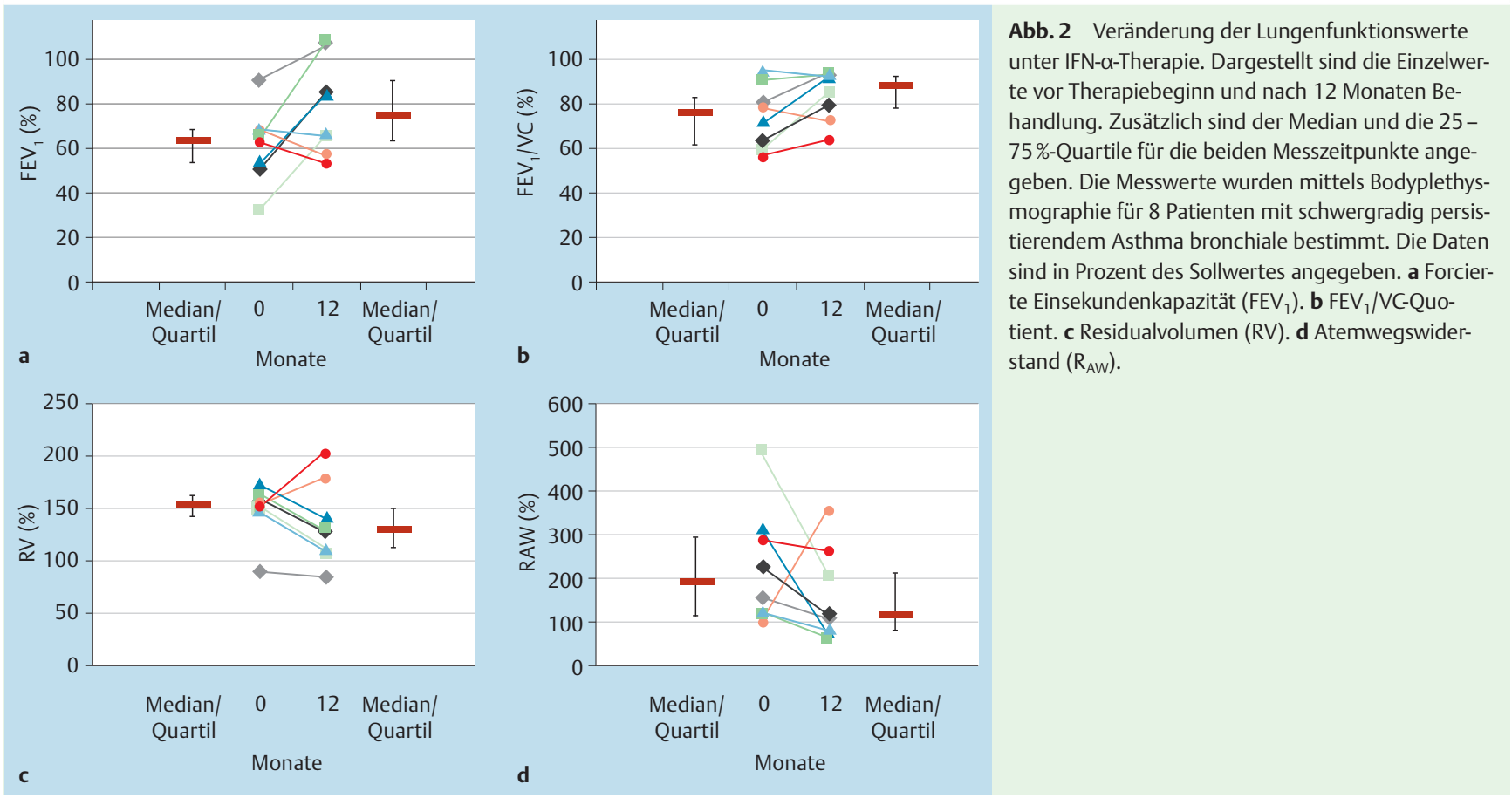




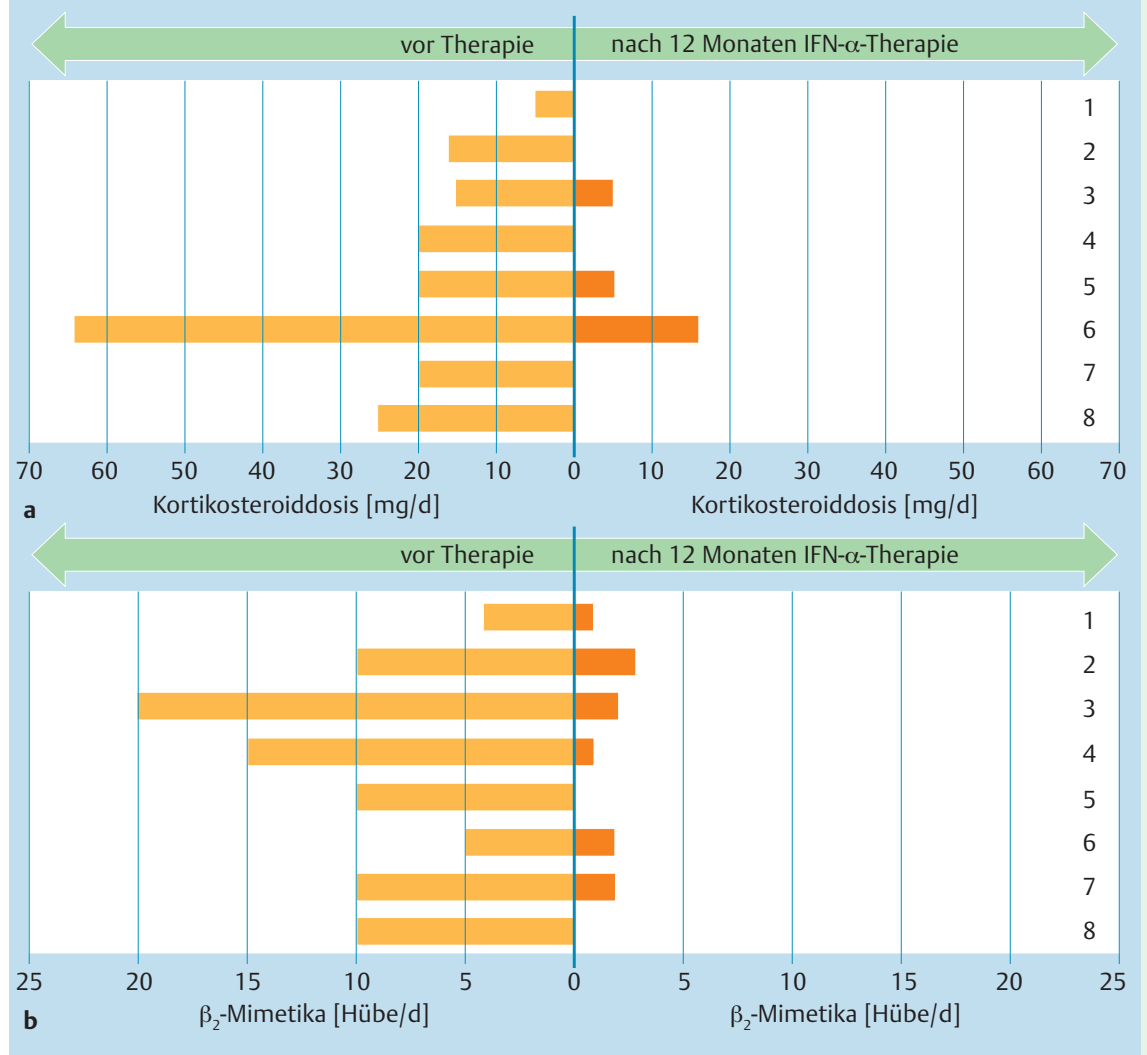

Abb. 3 Veränderung des Medikamentenbedarfs unter IFN- $\alpha$-Therapie. Die orale Glukokortikoiddosis (a) ist in $\mathrm{mg} / \mathrm{d}$ und die $\beta_{2}$-Mimetika Bedarfsmedikation (b) in Hübe/Tag von 8 Patienten mit schwergradig persistierendem Asthma bronchiale vor Therapiebeginn und nach 12 Monaten Behandlung dargestellt. Die Daten wurden anhand der Krankenakte der Patienten und des Patientenfragebogens ermittelt.

\section{Kurz wirksame $\beta_{2}$-Mimetika}

Der Einsatz von kurz wirksamen $\beta_{2}$-Mimetika konnte von 10,0 $(2,0-20,0)$ Hübe/Tag vor Therapie auf 1,0 $(0-3)$ Hübe/Tag nach 12 Monaten Therapie reduziert werden $(\mathrm{p}<0,05)$. Selbst Patienten $(3,4)$, die vor Therapiebeginn einen sehr hohen Bedarf angaben ( $>15$ Hübe/Tag), reduzierten diesen unter der Interferon- $\alpha$ Therapie auf $<5$ Hübe/Tag nach 12 Monaten $(\bullet$ Abb. $\mathbf{3 b}$ ).

\section{Zahl der eosinophilen Granulozyten}

Bis zum 6. Therapiemonat war eine Reduktion der Bluteosinophilenzahl im Median von 0,46 (0,05-0,74) Gpt/l auf 0,18 (0-0,41) $\mathrm{Gpt} / \mathrm{l}$ nachweisbar. Danach stieg die Bluteosinophilenzellzahl bei allen Patienten $(\mathrm{n}=8)$ wieder an. Nach 12-monatiger IFN- $\alpha$-Therapie lag der Median bei $0,28(0,06-0,99) \mathrm{Gpt} / \mathrm{l}$. Dies entspricht einer nicht signifikanten Abnahme der Eosinophilenzellzahl um 39,1\% gegenüber dem Median vor Therapie ( $\bullet$ Abb. 4 ).

\section{Diskussion}

Patienten mit schwergradig persistierendem Asthma bronchiale bleiben trotz Ausschöpfung aller Therapiemöglichkeiten symptomatisch. Alleine eine chronische orale Kortisontherapie erhält den Patienten am Leben. Adjuvante Medikamente, wie kolloidale Goldpräparate [4,5], Cyclosporin A [6,7], Cyklophosphamid oder Methotrexat $[8,9]$ und dem kortikosteroidsparenden Troleandomycin [10], zeigen keine einheitliche Wirkung und ein therapeutischer Effekt wurde bei längerer Anwendung bisher nicht nachgewiesen [11]. Darüber hinaus verursachen diese z.T. erheblichen Nebenwirkungen (Hepato- oder Nephropathie, Myeolotoxizität, (opportunistische) Infektionen und (selten) Pneumoniten $[12,13]$. Aus diesem Grund besteht ein Bedarf an alternativen, wirkungsvolleren Ansätzen zur Behandlung dieser Patienten.

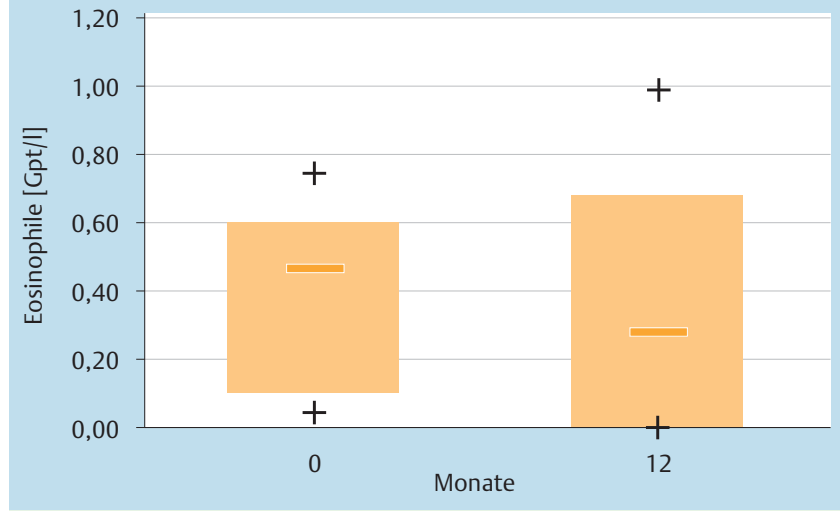

Abb. 4 Veränderung der Eosinophilenzellzahl unter IFN- $\alpha$-Therapie. Dargestellt ist der zeitliche Verlauf der Eosinophilenzahl über den Behandlungszeitraum von 12 Monaten. Abgebildet ist der direkte Vergleich der Mediane vor Therapie und nach 12 Monaten Behandlung. Zusätzlich sind die 25-75\%-Quartile für die Messzeitpunkte eingetragen. Die Eosinophilenzellzahl wurde durchflusszytometrisch ermittelt. Die Daten wurden für 8 Asthmatiker erfasst und sind in Gpt/l angegeben.

Die vorgelegte Studie zur Therapie von schwergradig persistierendem Asthma bronchiale beschreibt in einer offenen, fallkontrollierten Studie den Effekt von IFN- $\alpha$ auf schwergradig persistierendes Asthma bronchiale. Bei den 8 über 12 Monate behandelten Asthmatikern beeinflusste Interferon alle relevanten klinischen, lungenfunktionellen und laborchemischen Parameter. So nahm die Zahl der Asthmaattacken ebenso wie die Zahl der durch Asthma gestörten Nächte, die Häufigkeit der erforderlichen Notarztinterventionen und Hospitalisierungen ab. Bei fünf Asthmatikern ermöglichte IFN- $\alpha$ eine vollständige Kontrolle der Erkrankung ohne jegliche Asthmaanfälle am Tag oder in der Nacht. Zudem entwickelt sich eine anhaltende Verbesserung der Lungen- 
funktion mit Zunahme des PEF-Wertes, der $\mathrm{FEV}_{1}$, des Tiffenau-Indexes, Abnahme der RV und der Rtot sowie eine Zunahme des PEF um bis zu 190 L/min. Darüber hinaus verringerte sich der Bedarf an Beta-2-Mimetika/d deutlich, ebenso wie die Zahl der durch Asthma gestörten Nächte, die Häufigkeit der erforderlichen Notarztinterventionen und Hospitalisierungen. Gleichzeitig war es möglich, die orale Kortisondosis allmählich zu reduzieren. In fünf Fällen (62,5\%) konnte innerhalb von 6 bis 9 Monaten nach Therapiebeginn bei erhaltener Kontrolle die orale Kortisontherapie ganz beendet werden. Schließlich nahm auch die Bluteosinophilenzahl signifikant auf $0,28 \mathrm{Gpt} / \mathrm{L}$ ab, und zwar trotz Beendigung der oralen Kortisontherapie oder einer verminderten täglichen Kortisondosis.

Der therapeutische Einsatz von Interferonen, insbesondere Interferon- $\alpha$, hat sich in der Behandlung chronischer Virusinfektionen sowie maligner Erkrankungen etabliert, wie z.B. der chronischen Hepatitis B und C [14], verschiedenen Leukämieformen, beim malignen Melanom und dem Hypernephrom, endokrinen Pankreastumoren und dem Kaposi-Sarkom [14,15]. Darüber hinaus haben mehrere Untersuchungen der letzten Jahre gezeigt, dass IFN- $\alpha$ einen günstigen Effekt auf mit Eosinophilie assoziierten Erkrankungen wie dem Hypereosinophilen Syndrom und dem Churg-Strauss-Syndrom [16-24] besitzt. Kasuistiken beschreiben auch eine günstige therapeutische Wirkung von IFN- $\alpha$ bei steroidresistenten Asthmatikern [24-28]. Vergleichbare Ergebnisse zeigt eine Untersuchung an sieben Patienten mit kortikosteroidresistentem Asthma, die über mehr als 5 Monate mit IFN$\alpha$ behandelt wurden [26]. Die hier präsentierte offene Studie an acht Patienten mit schwerem Asthma bronchiale bestätigt und erweitert diese Beobachtungen. Sie belegt eine für diese schwerste Asthmamanifestation deutliche klinische Wirksamkeit des IFN- $\alpha$, die bei einigen Patienten nicht nur eine vollständige Kontrolle des Asthmas, sondern auch das Führen eines „normalen“, von Asthma unbeeinflussten Lebens ermöglicht. Anforderungen des täglichen Lebens, wie An- und Auskleiden, Zähneputzen, Einkaufen oder Treppensteigen werden unter Therapie mit IFN- $\alpha$ wieder bewältigt. Zwei der behandelten Asthmatiker waren in der Lage, unter IFN- $\alpha$-Therapie ihre berufliche Tätigkeit wieder aufzunehmen, von der sie zuvor frühberentet waren.

Seit kurzem steht mit dem Anti-IgE-Antikörper Omalizumab ein Medikament zur Verfügung, das für die Behandlung des schwersten Asthmas zugelassen wurde [28]. Die Therapie bietet bei eindeutig nachgewiesenem allergischen Asthma eine reale Therapieoption für diese Patienten, die über die Standardbehandlung hinausgeht [29]. Allerdings ist die Indikation auf ein allergisch verursachtes Asthma mit einem IgE-Spiegel zwischen 30 und 700 IE/L limitiert. Patienten außerhalb dieses Spektrums oder nicht-allergischen Asthmatikern steht Omalizumab zur Behandlung nicht zur Verfügung. Für diese Patienten, ebenso wie für solche, die nur unzureichend auf Omalizumab ansprechen, bietet die Behandlung mit Interferon eine Therapieoption.

Die wichtigste Einschränkung der IFN- $\alpha$-Therapie bei schwergradig persistierendem Asthma bildeten die Nebenwirkungen. Vier der ursprünglich in die Untersuchung eingeschlossenen Asthmatiker mussten die Therapie wegen depressiver Verstimmung abbrechen. Ein Patient lehnte die Behandlung nach der ersten Injektion ab. Bei den übrigen drei Patienten wurden die Kosten für die Therapie von der Krankenkasse nicht übernommen. Bei den über 12 Monate behandelten Patienten traten initial Fieber, Gelenkund Muskelschmerzen und Inappetenz 2 bis 12 Stunden nach der Interferoninjektion auf. Diese bildeten sich bei fünf Patienten nach dem ersten Behandlungsmonat zurück. Bei drei Patienten bestanden diese Nebenwirkungen fort und ließen sich durch eine Halbierung der Interferondosis beherrschen. Zudem kam es bei den Patienten zu einer leichten Thrombopenie, die aber konstant blieb und einen Wert von $100000 \mathrm{Gpt} / \mathrm{L}$ nicht unterschritt. Zwei Patienten entwickelten unter IFN- $\alpha$ eine Autoimmunthyreoiditis, die mit L-Thyroxin zu beherrschen war. Bemerkenswert war die Bereitschaft der Patienten, diese Nebenwirkung aufgrund nicht gekannter Kontrolle ihrer Erkrankung und des Zugewinns an Lebensqualität zu tragen. Die in Einzelfällen beschriebene Exazerbation eines vorbestehenden Asthma unter Interferontherapie bei chronischer Hepatitis $C$ oder idiopathischer Pulmonalfibrose $[30,31]$ wurde hier nicht beobachtet. Auch andere beschriebene Nebenwirkungen wie systemischer Lupus erythematodes (SLE), Raynaud-Syndrom, Autoimmunvaskulitis, rheumatoide Arthritis oder bullöses Pemphigoid entwickeln sich nicht. Alle IFN- $\alpha$-assoziierten Nebenwirkungen waren nach Absetzen der Therapie reversibel.

Bemerkenswert ist, dass sich viele der unter nicht-pegyliertem Standard-IFN- $\alpha$ beobachteten Nebenwirkungen einschließlich der Autoimmunthyreoditis unter pegyliertem IFN- $\alpha$ nicht mehr manifestierten [32]. Das lässt den Schluss zu, dass ein großer Teil der Nebenwirkungen offenbar durch die Spitzenkonzentration des nicht-pegylierten IFN- $\alpha$ hervorgerufen werden.

Der Mechanismus der anti-asthmatischen Wirkung ist derzeit noch nicht bis in alle Einzelheiten bekannt. Aufgrund seiner fördernden Wirkung auf Th1-Zellen und Hemmung der Th2-Zelldifferenzierung könnte die beim Asthma bestehende Th1/Th2-Imbalance korrigiert werden. Die Folge der reduzierten Th2-Zellaktivität unter IFN- $\alpha$ ist eine Reduktion der Eosinophilenzahl, der Abnahme des B-Zellanteils sowie der Verminderung der IgEProduktion unter Therapie (Daten nicht dargestellt).

Zusammengenommen zeigen die Ergebnisse, dass sich unter Therapie mit IFN- $\alpha$ bei Patienten mit schwergradig persistierendem Asthma bronchiale die Therapie mit systemischen Glukokortikoiden bei gleichzeitig verbesserter Kontrolle der Erkrankung reduzieren oder sogar beenden lässt. IFN- $\alpha$ bietet eine wirkungsvolle Alternative für Patienten mit schwergradig persistierendem Asthma, die die Indikation zur Omalizumabtherapie nicht erfüllen oder auf dieses Medikament nur unzureichend ansprechen.

\section{Interessenkonflikte}

$\nabla$

Keine angegeben

\section{Literatur}

1 Kroegel C, Reissig A, Mock B. Eosinophilic pneumonia. In: Gibson J, Geddes DM, Costabel U et al. (Hrsg). Respiratory Medicine. 3. Aufl. London/ New York: Harcourt Health Science, 2002

2 Weißbuch Lunge 2000. Defizite, Zukunftsperspektiven, Forschungsansätze: Zur Lage der Pneumologie in Deutschland. Stuttgart: Thieme, 2000

3 Sachverständigenrat für die Konzertierte Aktion im Gesundheitswesen. Gutachten 2000/2001 - Bedarfsgerechtigkeit und Wirtschaftlichkeit. Baden Baden: Nomos, 2002

4 Bernstein IL, Bernstein DI, Dubb JW et al. A placebo-controlled multicenter study of auranofin in the treatment of patients with corticosteroid-dependent asthma. Auranofin Multicenter Drug Trial. J Allergy Clin Immunol 1996; 98: 317-324

5 Evans DJ, Cullinan P, Geddes DM. Gold as an oral corticosteroid sparing agent in stable asthma. Cochrane Database Syst Rev 2001; CD002985

6 Nizankowska E, Soja J, Pinis G et al. Treatment of steroid-dependent bronchial asthma with cyclosporin. Eur Respir J 1995; 8: 1091 - 1099 
7 Evans DJ, Cullinan P, Geddes DM. Cyclosporin as an oral corticosteroid sparing agent in stable asthma. Cochrane Database Syst Rev 2001; CD002993

8 Erzurum SC, Leff JA, Cochran JE et al. Lack of benefit of methotrexate in severe, steroid-dependent asthma. A double-blind, placebo-controlled study. Ann Intern Med 1991; 114: 353 - 360

9 Davies H, Olson L, Gibson P. Methotrexate as a steroid sparing agent for asthma in adults. Cochrane Database Syst Rev 2000; CD000391

10 Evans DJ, Cullinan P, Geddes DM. Troleandomycin as an oral corticosteroid steroid sparing agent in stable asthma. Cochrane Database Syst Rev 2001; CD002987

11 Seelbach H, Ehmann R, Simon HU et al. Interferon-alpha beim steroidresistenten Asthma bronchiale und Churg-Strauss-Syndrom. Atemw Lungenkrkh 2000; 26: 354-355

12 Chumbley LC, Harrison EGJ, DeRemee RA. Allergic granulomatosis and angiitis (Churg-Strauss syndrome). Report and analysis of 30 cases. Mayo Clin Proc 1977; 52: 477-484

13 Lhote F, Guillevin L. Polyarteritis nodosa, microscopic polyangiitis and Churg-Strauss syndrome. Sem Respir Crit Care Med 1998; 19: 27 - 45

14 Kraus MR, Wilms K. Interferon-alpha Wirkung, Indikationen, Therapieüberwachung und Nebenwirkungen. Internist (Berl) 2000; 41: 1399 1404

15 Antoniou KM, Ferdoutsis E, Bouros D. Interferons and their application in the diseases of the lung. Chest 2003; 123: 209-216

16 Mock B, Bartuschka B, Kroegel C et al. Behandlung eines steroidresistenten Churg-Strauss-Syndroms mit Interferon-gamma. Atemw Lungenkrkh 1997; 23: $552-557$

17 Aldebert D, Lamkhioued B, Desaint $C$ et al. Eosinophils express a functional receptor for interferon alpha: inhibitory role of interferon alpha on the release of mediators. Blood 1996; 87: 2354-2360

18 Tatsis E, Schnabel A, Gross WL. Interferon-alpha treatment of four patients with the Churg-Strauss syndrome. Ann Intern Med 1998; 129: $370-374$

19 Reissig A, Forster M, Mock B et al. Behandlung des Churg-Strauss-Syndromes mit Interferon-alpha. Dtsch Med Wochenschr 2003; 128: $1475-1478$
20 Murphy PT, Fennelly DF, Stuart M et al. Alfa-interferon in a case of hypereosinophilic syndrome. Br J Haematol 1990; 75: 619-620

21 Zielinski RM, Lawrence WD. Interferon-alpha for the hypereosinophilic syndrome. Ann Intern Med 1990; 113: 716 - 718

22 Busch FW, Schmidt H, Steinke B. Alpha-interferon for the hypereosinophilic syndrome. Ann Intern Med 1991; 114: 338 - 339

23 Butterfield $J H$, Gleich GJ. Interferon-alpha treatment of six patients with the idiopathic hypereosinophilic syndrome. Ann Intern Med 1994; 121: $648-653$

24 Haefner D, Foerster M, Hengst $U$ et al. Immunmodulatorische Behandlung des schweren Kortikosteroid-abhängigen Asthma bronchiale und des Churg-Strauss-Syndroms mit Interferon-a. Klinischer Verlauf. Pneumologie 2001; 55: 71 [Abstract]

25 Gratzl S, Palca A, Schmitz M et al. Treatment with IFN- $\alpha$ in corticosteroid-unresponsive asthma. J Allergy Clin Immunol 2000; 105: 1035 1036

26 Simon HU, Seelbach H, Ehmann R et al. Clinical and immunological effects of low-dose IFN- $\alpha$ treatment in patients with corticosteroid-resistant asthma. Allergy 2003; 58: 1250-1255

27 Kroegel C. Asthmatherapie. Leitfaden einer pathogenetisch begründeten Therapie. 2. Aufl. Steinen: ZETT, 2005

28 Kroegel C, Bergmann N, Foerster $M$ et al. Interferon-alphacon-1 treatment of three patients with severe glucocorticoid-dependent asthma. Effect on disease control and systemic glucocorticosteroid dose. Respiration 2006; 73: 566 - 570

29 Kroegel C, Foerster M. Omalizumab. In: Dübel S (Hrsg). Handbook of Therapeutic Antibodies. 1. Aufl. Weinheim: Wiley-VCH, 2007: 951 997

30 Bini EJ, Weinshel EH. Severe exacerbation of asthma: a new side effect of interferon-alpha in patients with asthma and chronic hepatitis $C$ Mayo Clin Proc 1999; 74: 367 - 370

31 Honore I, Nunes H, Groussard 0 et al. Acute respiratory failure after interferon-gamma therapy of end-stage pulmonary fibrosis. Am J Respir Crit Care Med 2003; 167: 953 -957

32 Heider C, Förster M, Bergmann $N$ et al. Schwergradig persistierendes unkontrollierbares Asthma bronchiale. Eine Fall-kontrollierte Studie zur Therapie mit Interferon. Pneumologie 2009; 63 [V236]: 11 